\title{
La homonimia en diccionarios pedagógicos
}

A homonímia em dicionários pedagógicos

\author{
Renato RODRIGUES-PEREIRA* \\ Universidade Federal de Mato Grosso do Sul (UFMS)
}

\begin{abstract}
RESUMEN: En este artículo presentamos reflexiones sobre la homonimia en la Lexicografía Pedagógica (LEXPED), como forma de demostrar la pertinencia de criterios de naturaleza sincrónica para establecer unidades léxicas homónimas que compongan la macroestructura de diccionarios pedagógicos. Para ello, discurrimos respecto al origen de la LEXPED, así como exponemos lo que defendemos por "diccionarios didácticos" o "diccionarios organizados de forma más didáctica"; realizamos un breve repaso bibliográfico sobre la homonimia en diccionarios, según las posiciones de estudiosos como Fernandez-Sevilla (1974), Werner (1982), Biderman (1991), Clavería y Planas (2001), Porto Dapena (2002), Castillo Carballo (2003), Zavaglia (2011), y Pereira (2018); y por último, damos a conocer lo que Pereira (2018) y Pereira; Nadin (2020) han desarrollado en términos de investigación semántica con vistas al establecimiento de homonimia destinada a diccionarios pedagógicos. Como principios teóricos y metodológicos, nos orientamos por los de la LEXPED y de la Semántica léxica.
\end{abstract}

PALABRAS CLAVE: Léxico. Homonimia. Lexicografía Pedagógica.

RESUMO: Com este artigo, apresentamos reflexões sobre a homonímia na Lexicografia Pedagógica (LEXPED), com o propósito de demonstrar a pertinência de critérios de natureza sincrônica para o estabelecimento de unidades léxicas homônimas que venham a compor a macroestrutura de dicionários pedagógicos. Para tanto, discorremos a respeito da origem da LEXPED, assim como expomos o que defendemos por "dicionários didáticos" ou "dicionários organizados de forma mais didática"; realizamos uma breve revisão bibliográfica sobre a homonímia em dicionários, segundo posicionamentos de estudiosos como Fernandez-Sevilla (1974), Werner (1982), Biderman (1991), Clavería y Planas (2001), Porto Dapena (2002), Castillo Carballo (2003), Zavaglia (2011) e Pereira (2018); e por último, demonstramos o que Pereira (2018) e Pereira; Nadin (2020) tem desenvolvido a partir de pesquisa semântica com vistas à delimitação de homonímia destinada a dicionários pedagógicos. Como princípios teóricos e metodológicos, nos orientamos pelos da LEXPED e da Semântica léxica.

PALAVRAS-CHAVES: Léxico. Homonímia. Lexicografia Pedagógica.

\section{Introducción}

Las reflexiones sobre el establecimiento de la homonimia, específicamente destinada a diccionarios, han suscitado algunas discusiones en el mundo académico, como pretendemos demostrar con este artículo. Las Unidades Léxicas Homónimas (ULH) de una lengua, sea materna o extranjera, como pertenecientes al léxico general, reflexionan diversos aspectos de una comunidad socio-lingüístico-cultural. Por ello, una

\footnotetext{
* Doctor en Lingüística y Lengua Portuguesa por la UNESP/Araraquara/SP. Docente del Curso de Letras Portugués/Español y del Programa de Posgrado en Letras, maestría y doctorado, del Campus de Três Lagoas de la Universidade Federal de Mato Grosso do Sul. E-mail: renato.r.pereira@ufms.b74
} 
atención especial a esta parcela del léxico en diccionarios pedagógicos se vuelve necesaria, a medida que pensamos y elaboramos materiales didácticos dentro de una perspectiva de enseñanza/aprendizaje de lenguas en que el léxico ocupa un papel de destaque. Además, lo que diferencia las ULH en términos de importancia o atención en lo que se refiere a su registro en repertorios lexicográficos pedagógicos es justamente sus valores semánticos en relación a las otras Unidades Léxicas (UL) de la lengua, así como sus formas ortográficas y pronunciación semejantes.

En este contexto, tenemos UL que poseen valores semánticos distintos y misma grafía, como en los casos de homófonos homógrafos a seguir: i) gato: "s. 1 mamífero felino y carnicero, doméstico, de cabeza redonda, lengua muy áspera y pelo espeso y suave, que es muy hábil cazando ratones $[\ldots]$ ” $\neq$ gato: “3 Máquina compuesta de un engranaje, que sirve para levantar grandes pesos a poca altura [...]” (GONZÁLEZ, 2005, p. 612); o ii) llama: "s.f. 1 Masa gaseosa que arde y se eleva desprendiendo luz y calor [...]" \# llama: "3 (macho/hembra) Mamífero rumiante con pelo de color marrón claro y orejas largas y erguidas, que se utiliza como animal de carga y del que se obtiene leche, carne y lana [...]” (GONZÁLES, 2005, p. 871) = llama: “v. 1 tercera persona de presente de indicativo del verbo llamar [...]" o "2 Modo imperativo de segunda persona del verbo llamar [...]" (PEREIRA, 2,18, p. 174).

Además, hay lexías que poseen grafía y significados diferentes, pero con una misma pronunciación, a ejemplo de los homófonos no homógrafos ejemplificados en la secuencia: i) tubo/tuvo: "s.m. 1 Pieza hueca, de forma generalmente cilíndrica, que suele estar abierta por los dos extremos [...]" (GONZÁLES, 2005, p. 1356) y “v. irreg. Tercera persona de pretérito perfecto simple del verbo tener [...]" (PEREIRA, 2018, p. 178), respectivamente; o, ii) baca/vaca: "s.f. En un automóvil, soporte que se coloca sobre el techo y que sirve para llevar bultos [...]" (GONZÁLEZ, 2005, p. 153) y "s.f. 1 Hembra del toro [...]" (GONZÁLEZ, 2005, p. 1367), respectivamente. Esas ULH ni siempre suelen recibir tratamientos acordes a sus características funcionales $\mathrm{y}$ pragmáticas en los repertorios lexicográficos pedagógicos, como lo hemos percibido y demostrado en Pereira (2018, p. 126-166) ${ }^{1}$.

\footnotetext{
${ }^{1}$ En la oportunidad, fueron analizados un total de 20 diccionarios con el objetivo de verificar: i) si los diferentes tipos de homónimos hacen parte de la macroestructura de los diversos tipos de diccionarios; ii) si las distintas formas de tratamiento de homónimos en diccionarios están de acuerdo con las necesidades 
En efecto, el tratamiento lexicográfico de ULH es un hecho que hemos juzgado merecer atención especial, sobre todo en el ámbito de la Lexicografía Pedagógica (LEXPED), vertiente de la Lexicografía General, que se ocupa de estudios relacionados a la elaboración y al uso de diccionarios dirigidos a estudiantes de lengua materna o aprendices de lengua extranjera, como hemos explicado en la sección 1 de este texto.

En este escenario, con este artículo, presentamos algunas reflexiones sobre la homonimia en diccionarios. Para ello, objetivamos: i) hacer un breve repaso sobre el origen de la LEXPED, así como lo que defendemos por "diccionarios didácticos" o “diccionarios organizados de forma más didáctica"; ii) realizar una breve revisión bibliográfico sobre la homonimia en diccionarios, según las posiciones de estudiosos como Fernandez-Sevilla (1974), Werner (1982), Biderman (1991), Clavería y Planas (2001), Porto Dapena (2002), Castillo Carballo (2003), Zavaglia (2011), y Pereira (2018); iii) exponer lo que Pereira (2018) y Pereira y Nadin (2020) han desarrollado en términos de investigación semántica con vistas al establecimiento de homonimia destinada a diccionarios pedagógicos.

Para tanto, nos orientamos por principios teóricos y metodológicos de la LEXPED y de la Semántica léxica, con el objetivo de garantizar un trabajo con respaldos consistentes y que pueda significar una contribución para un área que mucho ha evolucionado, los Estudios del Léxico.

\section{Lexicografía Pedagógica}

Los estudios en el ámbito de la LEXPED, como los que mencionamos en la secuencia de este texto, nos informan que los diccionarios para aprendices de lenguas extranjeras surgieron en contextos de enseñanza, en que repertorios léxicos fueron organizados de acuerdo con las necesidades de los estudiantes en sus diferentes niveles de competencia en la lengua; y también a partir de los avances pedagógicos, con las nuevas tendencias didácticas que han revolucionado la enseñanza de lenguas desde el inicio del siglo $\mathrm{XX}^{2}$.

En Hartmann (1983), encontramos una primera reflexión sobre el diccionario en la enseñanza de lenguas, cuando Samuel Johnson (1747) dijo que "el valor del trabajo

\footnotetext{
${ }^{2}$ Respecto a este asunto, sugerimos conferir Molina (2006); Sánchez Ramos (2003); Welker (2008); y Rundell (1998).
} 
lexicográfico debe de ser estimado en el uso: que el diccionario satisfaga solo a los críticos no es suficiente, a menos que instruya/enseñe al usuario" (JOHNSON, 1747, apud HARTMANN, 1983, p. 9, traducción nuestra - $\mathrm{TN}^{3}$ ). Pero es a comienzos del siglo XX que tenemos, de acuerdo con Molina (2006, p. 9-21), el inicio de una labor lexicográfica con fines pedagógicos.

A lo largo del siglo XX, hubo una gran producción de diccionarios con fines pedagógicos. En ese período, los estudiosos considerados más importantes por sus trabajos en esa área de investigación son los lingüistas M. West, H. E. Palmer e A. S. Hornby, todos profesores de inglés, más específicamente en India el primero, y Japón el segundo y el tercero. Ellos pertenecían a programas de investigación sobre la enseñanza de lengua extranjera en que la producción de un diccionario para estudiantes no era precisamente el objetivo final de sus estudios. Pero acabaron por iniciar un trabajo lexicográfico que mucho ha servido de estímulo para otros investigadores y profesores.

De acuerdo con Molina (2006), el objetivo principal de Palmar era el de facilitar el proceso de aprendizaje de estudiantes de inglés como lengua extranjera (ILE) por medio de un vocabulario limitado, en que se seleccionaban como UL aquellas que eran más usuales en la comunicación diaria y que pudieran servir como materia léxico en las definiciones de los lemas. La idea de utilizar un vocabulario limitado se refiere al hecho de hacer que las definiciones de los diccionarios sean lo más claras y precisas posibles.

Así como Palmer, West también se interesó por investigar sobre un vocabulario limitado. Él trabajaba en la India compilando lecturas simplificadas para los estudiantes, como forma de recoger un número de palabras indispensables en la enseñanza de la lengua extranjera.

West y Endicott, de acuerdo con Sánchez Ramos (2003, p. 144), “consiguieron fijar un vocabulario limitado de 1490 palabras para definir 23.898 unidades léxicas en su diccionario The New Method English Dictionary (1935)". De considerable importancia para la Lexicografía, la obra sentó las bases para futuros repertorios léxicos que serían utilizados en las definiciones de las distintas entradas de diccionario. En realidad, constituyó el origen del vocabulario controlado en la primera edición del diccionario monolingüe de Longman, de 1978.

\footnotetext{
3 "The value of a work must be estimated by its use: it is not enough that a dictionary delights the critic, unless at the same time it instructs the learner" (JOHNSON, 1747, apud HARTMANN, 1983, p. 9). 
El primer repertorio con fines pedagógicos que se conoce es el Thousand-word Wnglish, una compilación de A. S. Hornby, en 1937. De acuerdo con Sánchez Ramos (2003, p. 145), las entradas eran lexías que aparecían de la misma forma que en los diccionarios que tenemos en la actualidad. Las flexiones y formas derivadas, por ejemplo, no eran registradas como entradas separadas, y sí aparecían como informaciones adicionales al lema.

Un año después, esa lista fue refinada por H. Palmer para la elaboración de su diccionario monolingüe, A Grammar of English Words, en 1938. La estructura de las entradas era semejante, aunque en esta nueva publicación eran incluidos ejemplos y frases idiomáticas.

Otra contribución para la LEXPED fue la de A. S. Hornby, con la publicación, en Japón, del diccionario Idiomactic and Syntactic English Dictionary (1942), que por las sucesivas ediciones pasó a ser titulado con las siguientes denominaciones: $A$ Learner's dicionary of Current English en 1948, The Advanced Learner's Dictionary of Current English en 1963, y el Oxford Advanced Learner's Dictionary of Current English en 1974.

En los años 70 del siglo XX, otros diccionarios continuaron esa tradición de los diccionarios pedagógicos, a ejemplo del Logman Dictionary of Contemporary English y del Chambers Universal Learner's Dicitonary, etc. Ya en los años 80, podemos encontrar diccionarios con semejantes características, como el Cambridge International Dictionary of English (1995), entre otros.

La producción de diccionarios elaborados con el objetivo de atender a necesidades de alumnos y profesores en la enseñanza y en el aprendizaje de lenguas, según Molina (2016), se quedó establecida por los tres autores ya mencionados: i) M. West, con el New Method English Dictionary, de 1935; ii) H. E. Palmer, con A Grammar of English Word, de 1938; iv) A. S. Hornby, con el Idiomatic and Syntactic English dictionary, con fecha de 1942.

Esos estudiosos, en efecto, al posibilitar la elaboración de diccionarios pedagógicos y contribuir, consecuentemente, con la enseñanza de inglés en el extranjero, propusieron una serie de principios que con el tiempo adquirieron la categoría de convención en el momento de plantear un diccionario. Tales principios: i) control de vocabulario; ii) información gramatical y sintáctica; iii) papel de los ejemplo; 
y iv) fraseología, sirvieron de guía para nuevas miradas lexicográficas con el fin de atender necesidades de los posibles usuarios, estudiantes de lenguas.

En países como Alemania, España, Francia, Brasil y Portugal, por ejemplo, hubo una considerable evolución en lo que se refiere a la LEXPED, aunque con menor o mayor cantidad, como podemos averiguar a partir de los diversos estudios sobre el área. En los diferentes contextos, lo que podemos verificar en términos de semejanza es que, por un lado, los estudios se vuelven a reflexiones metalexicográficas con objetivos de proponer parámetros que permiten repertorios elaborados más en conformidad con los principios de la LEXPED; por otro, los estudios se relacionan con el uso de los diccionarios pedagógicos en escenarios de enseñanza y de aprendizaje de lenguas.

En este contexto, Welker (2008, p. 15) se nos permite la asertiva de que la LEXPED se divide en dos campos: i) la teórica, que se ocupa de todo estudio que se vuelve para diccionarios pedagógicos; y ii) la práctica, responsable por la producción de estos diccionarios. De acuerdo con el autor, esas obras se diferencian de los diccionarios comunes por la preocupación con los aprendices, tanto el de lengua materna como el de lengua extranjera $\mathrm{y}$, por consiguiente, se considera las habilidades y necesidades de ellos.

Efectivamente, los diccionarios pensados y elaborados en el ámbito de los estudios teóricos y prácticos de la LEXPED permiten que los profesores y estudiantes tengan acceso a instrumentos pedagógicos que mucho contribuyen en la enseñanza de una lengua, puesto que posibilitan conocimientos de diferentes órdenes que, generalmente, son organizados de forma didáctica y, por ello, permiten al consultante, informaciones aclaradoras, en una perspectiva funcional-pragmática.

Los diccionarios pedagógicos, si son planteados de esa forma, adquieren particularidades informáticas sobre la/s lengua/s en estudio que, dependiendo de los procedimientos metodológicos adoptados por el profesor en sala de clase, o de actividad que el aprendiz esté realizando, la obra puede servir como un eficaz instrumento de consulta en el proceso de enseñanza y de aprendizaje.

En este contexto, resaltamos que no es pertinente que se predique toda obra lexicográfica como didáctica, pues no todas son organizadas con tal característica. Aunque en los últimos años muchos estudios relacionados a diccionarios pedagógicos han sido realizados y consecuentemente, proyectos de diccionarios de esa naturaleza 
han surgido cada vez más, no podemos olvidar que la mayoría de las obras lexicográficas existentes aún no es "didáctica", es decir, no es organizada de forma didáctica. Por ello, decir que todo diccionario es esencialmente didáctico es como si siguiéramos por caminos opuestos a las epistemologías relacionadas a la enseñanza, pues el término "didáctico/a" se nos remite a toda y a cualquier forma de ensañar a alguien. Destacamos pues: el diccionario no enseña, sino que proporciona informaciones lingüísticas y extralingüísticas que, solamente dependiendo de la capacidad de abstracción del consultante podrá ser aprovechado en toda su amplitud (PEREIRA, 2018).

Si acudimos a Cunha (2010, p. 218), tenemos: "didá(c)tica sf. 'ciência de ensinas' 1844. Do fr. didactique, deriv. do lat. med. didáctica (ars) e, este, do gr. didaktiké, fem. do adj. didaktikós, da raíz, didak-, de didáskein 'ensinar'// didá(c)tico 1844". Por la definición presentada, se puede notar que el término "didáctico/a" puede tener función tanto de sustantivo, designando una ciencia, una disciplina, como también tener la función de adjetivo, caracterizando alguna cosa, persona o situación.

En realidad, para que definamos un diccionario como didáctico, precisamos considerar: i) los estudios que son realizados en el ámbito de ciencias que poseen interfaces teórico-aplicadas, como la LEXPED, la Pedagogía y la Lingüística Aplicada a la Enseñanza de Lenguas; ii) el proceso de elaboración de la obra lexicográfica, de forma que, con el resultado, el estudiante pueda buscar informaciones y no necesite realizar otras pesquisas para poder sanar la duda en cuestión.

En este contexto, reflexiones como las que presentamos en la secuencia son muy pertinentes a nuestro juicio: ¿Las informaciones son claras, de fácil acceso?; ¿Las definiciones están elaboradas en una variedad de lengua adecuada a la edad del estudiante y de acuerdo con el nivel escolar?; ¿Cuál es el objetivo de la obra? Esas y otras cuestiones que vengan a posibilitar una mejora de los repertorios lexicográficos pedagógicos necesitan ser consideradas antes que prediquemos un diccionario como didáctico.

Para Krieger (2011, p. 106) dos son los principios esenciales en el ámbito de la LEXPED: i) la búsqueda de la adecuación del diccionario; y ii) el uso productivo para los distintos proyectos de enseñanza y aprendizaje de lenguas. La autora nos plantea aún que a los dos ítems deben ser agregados 
la comprensión de que el diccionario es un texto, con reglas propias de organización, que sistematiza inúmeras informaciones de carácter lingüístico, cultural y pragmático. De ello resulta su exponente papel pedagógico, bien como el principio de que así como hay libros didácticos adecuados a los diferentes niveles de enseñanza, de igual modo, se debe proceder a la escoja del diccionario adecuado a las necesidades de aprendizaje de los alumnos (KRIEGER, 2011, p. 106, traducción nuestra - TN) ${ }^{4}$.

Welker (2011), al discurrir también sobre los principios que direccionan la LEXPED, enfatiza que lo fundamental en la elaboración de diccionarios pedagógicos resulta de la consideración de las reales necesidades y habilidades de los usuarios. Para el autor, hay que considerar que la producción de diccionarios para aprendices debe acontecer de forma distinta, de acuerdo con los niveles de competencia lingüística que ellos se encuentran.

En suma, lo que se puede verificar es que los estudios realizados en el ámbito de la Lexicografía surgieron de la necesidad, en el campo de la enseñanza de lenguas, de diccionarios elaborados para públicos bien delimitados: estudiantes de lenguas extranjeras en sus diferentes niveles de competencia en el idioma. De ese modo, toda y cualquier investigación que tiene como objetivo propiciar reflexiones y parámetros que auxilien en la producción de diccionarios pedagógicos organizados de forma cada vez más didáctica siempre es bienvenida. En ese entorno, a partir de la próxima sección, presentamos un poco de lo que hemos leído e investigado sobre la homonimia en diccionarios pedagógicos.

\section{La homonimia en diccionarios: algunos posicionamientos}

La literatura que versa sobre la homonimia en diccionarios en una perspectiva pedagógica, por lo que hemos investigado, aún parece ser poco productiva en el medio académico (PEREIRA, 2018). En algunos casos, encontramos posturas teóricas en que se resalta que la solución es no hacer ninguna distinción entre unidades homónimas y polisémicas, de forma que en un mismo artículo, todos los significados sean agrupados (WERNER, 1982). El autor enfatiza aún que la aplicación del criterio etimológico

\footnotetext{
${ }^{4}$ a compreensão de que o dicionário é um texto, com regras próprias de organização, que sistematiza inúmeras informações de caráter linguístico, cultural e pragmático. Daí resulta seu exponencial papel pedagógico, bem como o princípio de que assim como há livros didáticos adequados aos diferentes níveis de ensino, de igual modo, deve-se proceder à escolha do dicionário adequado às necessidades de aprendizagem dos alunos (KRIEGER, 2011, p. 106).
} 
resuelve la necesidad práctica de los lexicógrafos cuando se deparan con la necesidad de recurrir a diferentes criterios para la definición y la ordenación de esas lexías.

Si seguimos ese entendimiento y pensamos la problemática considerando las múltiples posibilidades de criterios de definición de homonimia, y también de polisemia, en el ámbito de la Lexicología o de la Lingüística Cognitiva, por ejemplo, tal vez podamos nos desanimar delante de los variados e importantes caminos para la reflexión sobre el hecho lingüístico.

No obstante, si estamos delante del contexto específico de la LEXPED, podemos optar por un camino que esté más de acuerdo con las necesidades del público a que se destina los repertorios lexicográficos pedagógicos. En ese escenario, los enfoques sincrónicos parecen ser más adecuados, puesto que, para el estudiante, lo que le importa son los significados que una unidad léxica posee en el momento de la consulta.

Biderman (1991, p. 183-290), por ejemplo, al discurrir sobre la homonimia y la polisemia, destaca que "la significación actual del lexema es lo que interesa de hecho para el hablante" (p. 287, TN ${ }^{5}$ ). La investigadora, lexicógrafa brasileña, aclara que la historia de la palabra, en una perspectiva diacrónica, poco interesa a la mayoría de los hablantes, principalmente para los consultantes de un diccionario, aprendices de lengua. De ese modo, se queda manifestada su preferencia por el establecimiento de homonimia y de polisemia adoptando el criterio semántico, en una perspectiva sincrónica.

Clavería y Planas (2001, p. 281-306) hacen un importante análisis de diccionarios de lengua española, cuyo objetivo principal es profundizar los estudios relacionados al tratamiento de los homógrafos en la lexicografía española y con ello, aumentar el corpus de diccionarios analizados. Con el estudio, las autoras consideran especialmente el criterio semántico, justificando que en las investigaciones anteriores esta posibilidad de estudio había sido postergada.

También justifican que "el análisis contrastiva de varios diccionarios servirá, además, como punto de partida para reflexiones sobre la función de la homonimia en la Lexicografía y sobre cuáles son los presupuestos que deben ser considerados para su establecimiento" (CLAVERÍA; PLANAS, 2001, p. 282). Considerando los criterios gramatical, etimológico y semántico, las investigadoras analizan siete diccionarios con

\footnotetext{
5 “a significação atual do lexema é o que interessa à maioria dos falantes”(BIDERMAN, 1991, p. 287). 
el objetivo de observar la significación y el empleo de la homonimia en la lexicografía española.

Con los análisis realizados, se percibe que la aplicación del criterio semántico abre nuevas perspectivas de organización de homógrafos y acepciones, aumentando también la diversidad de soluciones. Para las autoras, el criterio semántico presenta una cuestión teórica fundamental que remite a cuestionamientos relacionados al establecimiento de cómo distinguir los diferentes significados a partir de un significado básico.

Ellas sugieren en este contexto una mirada hacia la semántica funcional y su posible aplicación en la Lexicografía. Aunque haya dificultades en la aplicación del criterio semántico y la variación generada en la cuestión analizada, las autoras enfatizan que los diccionarios DEUM - Diccionario del español usual de México, de Lara (1996), y el DEA - Diccionario del español actual, de Seco y Ramos (1999) abren nuevas perspectivas para la Lexicografía española con la incorporación de este criterio para el establecimiento de la macroestructura del diccionario, así como la posibilidad de una mejor forma de comprender y reflexionar la lengua.

Porto Dapena (2002), al reflexionar sobre el asunto, resalta la cuestión de la lematización y se posiciona diciendo que el problema se plantea solamente en el caso de los homógrafos, puesto que los homófonos ya son registrados de acuerdo con el orden alfabético que los diccionarios, en su macroestructura, suelen ser organizados. Para los homógrafos, el investigador se posiciona a favor de registrarlos en entradas distintas, como palabras diferente que son. El autor aborda, además, la dificultad en la hora de distinguir los casos de homonimia y de polisemia que los lexicógrafos suelen tener al inventariar los candidatos a lemas de un diccionario. En esta perspectiva, enfatiza tres criterios que, aun siendo importantes, no siempre llevan a los mismos resultados, a saber: diacrónico o etimológico, sincrónico y mixto.

Al discurrir sobre el criterio diacrónico, Porto Dapena (2002) evidencia la falta de estudios etimológicos sobre el vocabulario y que por ello, lexicógrafos acaban recurriendo a informaciones que no siempre son seguras. Sin embargo, deja entrever en la secuencia del texto su preferencia por el criterio diacrónico, cuando se posiciona ser la opción sincrónica más problemática. Porto Dapena destaca que la adopción del criterio sincrónico, al que él dualiza "criterio del sentimiento lingüístico y criterio 
componencial o semántico", se presenta mayores problemas (PORTO DAPENA, 2002, p. 188), de forma que para el autor:

[...] nos parece que lo más práctico en Lexicografía es mantener la distinción en los términos tradicionales, esto es, basándonos en la perspectiva diacrónica, cuya adopción por cierto en un estudio descriptivo del léxico no tiene por qué ser inadecuada: solo desde la historia nos es dados constar, efectivamente, si en relación con un mismo soporte fónico ha habido una divergencia de significado o, por el contrario, una convergencia de significados [...] (PORNO DAPENA, 2002, p. 190).

Castillo Carballo (2003, p. 99-101), por su vez, hace una reflexión respecto a la problemática existente en relación a los diferentes criterios de organización de las unidades léxicas homónimas en la macroestructura y recuerda que el punto de vista diacrónico es lo que ha predominado en el tratamiento de la homonimia en diccionarios, pero que "ha sido puesto en entredicho, porque resulta incongruente que un repertorio sincrónico tenga en cuenta una lengua funcional anterior" (CASTILLO CARBALlO, 2003, p. 100). Se observa, con las palabras de la autora, su posicionamiento contrario a esa postura en la labor lexicográfica. Ella añade también que el origen incierto de algunas palabras confirma la aplicación problemática del criterio diacrónico y que, por ello, muchos estudiosos prefieren el criterio semántico, en una perspectiva sincrónica.

En Zavalia (2011), tenemos que el fenómeno de la homonimia y de la polisemia es un desafío para los lexicógrafos, pues su delimitación y organización en las obras lexicográficas necesitan siempre ser sustentadas por parámetros teóricos y prácticos bien definidos y limitados. La lexicógrafa resalta, en este contexto, que al potencial consultante,

le restará la simple tarea de saborear la diversidad lexical que el fenómeno de la homonimia es capaz de producir en una lengua natural, no se importando con su origen etimológico, pero se encantando con su diversidad semántica (ZAVAGLIA, 2011, p. 43, $\mathrm{TN})^{6}$.

Otros autores que también tratan la homonimia en la Lexicografía, por ejemplo de Fernández-Sevilla (1974), Murakawa (2011) y Tarp (2013), nos presentan

\footnotetext{
6 “restará a simples tarefa de saborear a diversidade lexical que o fenómeno da homonímia é capaz de produzir em uma língua natural, não se importando com sua origem etimológica, mas se encantando com sua diversidade semântica" (ZAVAGLIA, 2011, p. 43).
} 
importantes reflexiones, no obstante, no se posicionan, por lo menos en esos trabajos, respecto a cuál enfoque, diacrónico o sincrónico, se nos parecen más adecuado para el establecimiento de homonimia destinada a diccionarios.

Por nuestra parte, estamos en el camino de los investigadores mencionados anteriormente que son a favor de criterios de naturaleza sincrónica: Biderman (1991), Clavería y Planas (2001), Castillo Carvallo (2003) y Zavaglia (2011). Por lo expuesto, verificamos que los criterios diferenciadores entre homonimia y polisemia para la definición de los fenómenos para la Lexicografía, así como para el establecimiento de parámetros organizativos de esas unidades léxicas de la lengua en diccionarios aún necesitan más reflexiones y, consecuentemente, decisiones teóricas y metodológicas que puedan figurarse como orientadoras por lo menos para los tipos de diccionarios que exigen tal procedimiento, como los diccionarios pedagógicos ${ }^{7}$.

En la próxima sección de este artículo nos detenemos a presentar lo que hemos hecho en términos de investigación semántica empírica, cuyo objetivo principal ha sido demonstrar la relevancia de considerarse la perspectiva sincrónica para el establecimiento de homonimia en el ámbito de la LEXPED.

\section{La homonimia en diccionarios: del establecimiento de la nomenclatura}

Por lo presentado hacia el momento, se percibe que para la definición de homonimia, en términos generales, uno puede optar por criterios de naturaleza sincrónica o diacrónica. Como fue enfatizado en la sección anterior, somos defensores de criterios sincrónicos. Más específicamente, somos a favor de que se establezca una unidad léxica como una lexía homónima cuando no haya ninguna relación de sentido entre dos o más formas (PEREIRA, 2018). Pero para ello, nos respaldamos en principios teóricos y metodológicos de la semántica lexical, en que investigaciones son realizadas con vistas a verificar, por ejemplo, en qué medida dos o más significados de un mismo significante se alejan de tal forma que no haya ninguna relación de sentido entre ellos.

En Pereira (2018) y Pereira; Nadin (2019), realizamos una investigación empírica, dentro de una perspectiva sincrónica, con el objetivo principal de posibilitar

\footnotetext{
${ }^{7}$ A ese respecto, indicamos la lectura de la investigación de Pereira (2018, p. 73-193).
} 
bases teóricas y metodológicas para el inventario de candidatos a homónimos destinados a diccionarios pedagógicos. Para eso, nos basamos en el principio de la “divergencia semántica” (ULLMANN, 1964), en el criterio de la “conciencia lingüística de los usuarios" (WERNER, 1982), así como en el relato que Biderman (1991) nos brinda sobre la investigación empírica de Soares da Silva (1989).

Ullmann (1964, p. 364-374) presenta la comprensión del proceso generativo de unidades léxicas homónimas a partir de tres procesos: i) convergencia fonética; ii) divergencia semántica; e iii) influencia extranjera. Para este artículo, en conformidad con los objetivos establecidos, discurrimos sobre el proceso ii).

La divergencia semántica, de acuerdo con el autor, es provocada por el desarrollo de sentidos divergentes. Ullmann destaca que ese hecho ocurre "cuando dos o más significados de la misma palabra se separan de tal modo que no haya ninguna conexión evidente entre ellos", de forma que "la polisemia dará lugar a la homonimia y a la unidad de la palabra será destruida" (ULLMANN, 1964, p. 368). El autor enfatiza aún que esta forma de homonimia resulta de la réplica exacta de los también conocidos homónimos reinterpretados, que en una perspectiva sincrónica un significante opera en direcciones opuestas, aunque la diferencia de significado no sea muy grande, pues "el locutor moderno, desconocedor de etimologías, establecerá una relación entre ellos sobre bases puramente psicológicas" (ULLMANN, 1964, p. 340).

Werner (1982), por su vez, presenta algunos criterios para diferenciar la homonimia de la polisemia. Entre ellos, enfatizamos dos: i) criterio etimológico; ii) criterio da conciencia lingüística de los usuarios.

En i), para el autor, la homonimia ocurre cuando los diferentes contenidos se refieren a significantes iguales, siempre y cuando en sus orígenes hayan tenido significantes diferentes. La polisemia, por su vez, cuando distintos contenidos se refieren a significantes iguales, siempre y cuando, a partir de un punto de vista diacrónico tengan un origen idéntico. Tenemos, pues, casos de convergencia diacrónica en el plano de la expresión y divergencia en el plano del contenido, respectivamente.

En el criterio II, Werner resalta que existe la homonimia cuando el hablante no establece ninguna relación entre los diferentes contenidos de una única forma en el plano de la expresión y, de forma contraria, hay la polisemia cuando en la conciencia del hablante existe una relación entre los diferentes contenidos que pueden corresponder 
a solo una forma en el plano de la expresión. Pero para el autor, para una distinción científica entre homonimia y polisemia, tal criterio no es pertinente, es decir, es poco científico, puesto que no se puede determinar de forma objetiva lo que el hablante de una lengua puede o no establecer como relación a una determinada unidad lingüística. Siguiendo los presupuestos teóricos de la semántica estructural, Werner (1982) propone la identificación de elementos comunes de sememas para los casos de identidad en el plano de la expresión y, diferentemente, la divergencia en el plano del contenido. De ese modo, por un lado habría homonimia cuando en el plano del contenido, esos sememas no poseyeran ningún sema en común y, por otro habría polisemia cuando a una única forma correspondieran varios sememas que poseyeran por lo menos un semema en común, en el plano de la expresión.

Biderman (1984), al tratar de la homonimia y de la polisemia en una perspectiva sincrónica, los apunta como algunos de los problemas que influyen en la elaboración de obras lexicográficas, pues determinan cuestiones relacionadas a la extensión de la macroestructura del diccionario, así como los relacionados a la microestructura del diccionario. Por las palabras de la investigadora, se percibe que la tesis de la conciencia del hablante adquiere un lugar de destaque. Al justificar la práctica moderna, la autora aclara que:

En la moderna lexicografía, sobre todo aquella que se hace en Francia, el procedimiento ha sido considerar homónimas palabras de grafía idéntica (mismo significante) y significados muy distintos, a punto de ser difícil para el hablante identificar semas comunes a los dos o más homónimos (BIDERMAN, 1984, p. 143, TN) ${ }^{8}$.

En esta misma línea, Biderman (1991) hace referencia al trabajo de Soares da Silva (1989), que sometió veinticuatro hablantes de la lengua portuguesa, más específicamente estudiantes de Humanidades de la Universidade Católica de Braga, a pruebas de identificación de unidades ambiguas. Soares da Silva

[...] elaboró una serie de frases sobre 100 significantes problemáticos (sustantivos, adjetivos, verbos), enunciados esos dispuestos en pares. De esas 100 solo 13 son clasificadas como homónimas por el diccionario Aurelio. Esos sujetos examinaron los pares de frases y fueron señalando los grados de similitud en una escala de 0 a 4 , considerando la palabra clave en pauta. Tal escala destacaba: 1) ausencia total de semejanza de sentido (0) palabras homónimas; 2)

\footnotetext{
${ }^{8} \mathrm{Na}$ moderna lexicografia, sobretudo aquela que se faz na França, o procedimento tem sido considerar homônimas palavras de grafia idêntica (mesmo significante) e significados muito distintos, a ponto de ser difícil para o falante identificar semas comuns aos dois ou mais homônimos (BIDERMAN, 1984, p. 143). 
grados diversos de similitud semántica (1 a 4) palabras polisémicas. El resultado de la investigación mostró que hay un alto grado de acuerdo entre los hablantes en la discriminación de estas dos categorías: media de $78 \%$. Y nunca ocurrió un desacuerdo grande entre los hablantes, es decir: un acuerdo inferior a $50 \%$ (BIDERMAN, 1991, p. 288, TN9).

Biderman destaca también que la investigación realizada por Soares da Silva evidenció que la distinción entre polisemia y homonimia en nivel de teoría lingüística no solo es posible como necesaria, pues "los criterios teóricos propuestos presentan resultados adecuados a la realidad de estos fenómenos y su operatividad podrá y deberá ser experimentada por el lexicógrafo" (SOARES DA SILVA, 1989, p. 10-11, apud BIDERMAN, 1991, $\left.\mathrm{TN}^{10}\right)$.

Otra investigadora que también mucho ha estudiado sobre la homonimia es Zavaglia (2003), que al discurrir sobre el criterio etimológico, resalta la dificultad existente en escogerlo y todavía, que es bastante común entre los expertos la no aceptación de este criterio. La autora resalta que ese es el más utilizado para hacerse la distinción de una lexía homónima de una polisémica. No obstante, como en portugués las investigaciones etimológicas son escasas e insuficientes para que se pueda ofrecer seguridad y credibilidad a los estudios que se refieren al origen de una palabra, "se vuelve difícil adoptar como criterio básico de identificación de un ítem lexical, el estudio diacrónico" (ZAVAGLIA, 2003, p. 01, TN ${ }^{11}$ ). Aún según esta investigadora, lo que se espera es que se pese los puntos positivos y los negativos de cualquier parámetro adoptado en una investigación.

Quizás, en medio a los muchos criterios, así como a partir de las decisiones importantes que debemos tomar cuando se plantea el inventario de unidades léxicas homónimas para componer la nomenclatura de un diccionario, podamos pensar en “[...] contentarnos con el hecho de que el problema de la distinción entre homonimia y

\footnotetext{
${ }^{9}$ [...] elaborou uma série de frases sobre 100 significantes problemáticos (substantivos, adjetivos, verbos), sentenças essas dispostas em pares. Dessas 100 apenas 13 são classificadas como homônimas pelo Aurélio. Esses sujeitos examinaram os pares de frases e foram assinalando os graus de similaridade numa escala de 0 a 4, levando em consideração a palavra-chave em pauta. Tal escala marcava: 1) ausência total de semelhança de sentido (0) palavras homônimas; 2) graus diversos de similaridade semântica (1 a 4) palavras polissêmicas. $\mathrm{O}$ resultado da pesquisa mostrou que há um alto grau de acordo entre os falantes na discriminação destas duas categorias: média de 78\%. E nunca ocorreu um desacordo grande entre os falantes, ou seja: um acordo inferior a 50\% (BIDERMAN, 1991, p. 288).

10 "os critérios teóricos propostos apresentam resultados adequados à realidade destes fenômenos e a sua operacionalidade poderá e deverá ser experimentada pelo lexicógrafo" (SOARES DA SILVA, 1989, p. 10-11, apud BIDERMAN, 1991).

11 "torna-se difícil adotar como critério básico de identificação de um item lexical, o estudo diacrónico" (ZAVAGLIA, 2003, p. 01).
} 
polisemia sea en principio, irresoluble" (LYONS, 1987, p. 111, TN ${ }^{12}$ ). Sin embargo, así como Biderman (1984; 1991; 1998), por ejemplo, comprendemos que al estudiante de una lengua, sea ella materna o extranjera, los valores semánticos de una palabra son más productivos y pragmáticos en una perspectiva sincrónica, pues el aprendiz necesita resolver sus dudas relacionadas a los significados, $\mathrm{u}$ otra información de naturaleza lingüística, como de uso, por ejemplo, en el momento de la consulta (PEREIRA, 2018; PEREIRA; NADIN, 2019).

Como se comprende por lo demostrado en la sección dos y en esta que nos detenemos ahora, para estudiosos defensores de otros criterios que no los de naturaleza sincrónica, a ejemplo de Werner (1982) y Porto Dapena (2002), la divergencia semántica resulta en un impedimento para que se puedan rescatar los varios valores semánticos de homonimia y polisemia de una misma palabra. Nosotros, no obstante, respaldados por Soares da Silva (1989, apud Biderman, 1991); Biderman (1984, 1991, 1998), Zavaglia (2003), Pereira (2018) y Pereira; Nadin (2020), entre otros, demostramos que es posible que se delimite unidades léxicas como casos de homonimia o de polisemia en una perspectiva sincrónica.

En Pereira (2018) y Pereira y Nadin (2019), demostramos la investigación semántica empírica inicial ${ }^{13}$ que realizamos con vistas a demostrar que la perspectiva sincrónica para la definición de homonimia destinada a diccionario pedagógicos es perfectamente posible y pertinente en el ámbito de la LEXPED. Para ello, de acuerdo con el relato de Biderman (1991) sobre la investigación empírica de Soares da Silva, con el principio de la "divergencia semántica", de Ullmann (1984), y con la "conciencia lingüística de los usuarios", de Werner (1982), desarrollamos la investigación que tuvo como objetivo verificar en qué medida un hablante no insertado en los estudios lingüísticos de la lengua portuguesa es capaz de diferenciar una unidad léxica homónima de una polisémica.

Con la investigación, a partir de la aplicación de un cuestionario a un total de 119 informantes, pudimos constatar la pertinencia de considerar una UL como un caso

\footnotetext{
12 “[...] nos contentar como o fato de que o problema da distinção entre homonímia e polissemia seja, em princípio, insolúvel” (LYONS, 1987, p. 111).

${ }^{13}$ Actualmente, estamos realizando la investigación con hablante nativos de la lengua española, más específicamente con alumnos de la UNC - Universidad Nacional de Córdoba, a partir de la relación establecida entre esa universidad y la UFMS - Universidade Federal de Mato Grosso do Sul, vía Proyecto Lexicografia Pedagógica: elaboração do dicionário de formas homónimas em español para aprendizes brasileiros, desde nuestra coordinación.
} 
de homonimia, partiendo, pues, de principios teóricos y metodológicos de naturaleza sincrónica para su delimitación. Para tanto: i) escogimos aleatoriamente diez unidades léxicas que pueden ser consideradas formas homónimas homógrafas homófonas o polisémicas; ii) organizamos 21 pares de oraciones; iii) aplicamos el cuestionario al público mencionado en el párrafo anterior ${ }^{14}$. Después de la aplicación, en el momento de análisis de los datos obtenidos,

[...] consideramos casos de homonimia cuando, para la mayoría de los informantes, no hay ningún vínculo semántico entre las unidades léxicas, y polisémicas cuando, también para la mayoría, hay algún tipo de asociación" (PEREIRA, 2018, p. 81, TN ${ }^{15}$ ).

El resultado de la investigación semántica demostró un alto grado de acuerdo entre los hablantes investigados, con un promedio de $88 \%$. Así como en Soares da Silva (1989), no hubo en nuestra investigación un desacuerdo entre los hablantes. En nuestro caso, no hubo un desacuerdo inferior a $68 \%$, lo que para nosotros es un dato importante que puede ser verificado en secuencia de la investigación.

Por lo expuesto, se percibe que, en el escenario de la LEXPED, se puede establecer una lexía como un candidato a homónimo de un diccionario pedagógico a partir de la divergencia semántica existente entre dos o más significados. De acuerdo con el repaso bibliográfico demostrado en este artículo, en que pudimos visualizar posicionamientos autorales, así como investigaciones semánticas de naturaleza sincrónica, enfatizamos la pertinencia de considerar la homonimia sincrónica en el ámbito de la LEXPED.

\section{Consideraciones finales}

Estudiar el léxico en sus múltiples dominios permite vislumbrar diferentes y semejantes características de semantización. Cuando se trata de homonimia y polisemia, un significante ora adquiere valores semánticos completamente distintos, ora significados que se relacionan de alguna manera, independientemente del criterio adoptado por el investigador. No obstante, eso no significa que el investigador no tenga

\footnotetext{
${ }^{14}$ El público fue constituido por estudiantes de los primeros años de los cursos de grado de historia, matemáticas y geografía de una universidad pública.

15 “[...] consideramos casos de homonímia quando, para a maioria dos informantes, não há nenhum vínculo semântico entre as unidades léxicas, e polissêmicas quando, também para a maioria, há algum tipo de associação" (PEREIRA, 2018,p. 81).
} 
que escoger uno o más criterios que consigan posibilitarle datos seguros y pertinentes en relación a los objetivos que haya establecido.

Para investigaciones en el ámbito de la LEXPED, como lo hemos demostrado, cuanto más detallados sean los estudios teóricos y metodológicos que visan a propiciar al mundo académico diccionarios organizados de forma más didáctica, así como el uso productivo y efectivo de esa tipología de diccionarios, mejor serán los resultados de las investigaciones.

Considerando los objetivos de este texto, pudimos presentar, aunque brevemente, el origen de la LEXPED, así como resaltamos que no todos diccionarios son de hecho didácticos, pero que poseen potenciales didácticos, siempre y cuando sean organizados como tal.

Con el repaso bibliográfico realizados sobre la homonimia en diccionarios, resaltamos que los estudiosos no siempre comulgan con los mismos posicionamientos, lo que consideramos muy importante, sobre todo en el contexto de la ciencia, pues de esa forma se levantan problemas, hipótesis y se desarrollan nuevas investigaciones con fines de ampliar las reflexiones y contribuir con las ya existentes sobre un asunto.

En la exposición de la investigación semántica empírica inicial que hemos realizado, señalamos la posibilidad de considerarse la divergencia semántica en una perspectiva sincrónica para el establecimiento de ULH que vengan a formar parte de la macroestructura de diccionarios pedagógicos.

De ese modo, esperamos que el producto de este texto sirva de inspiración a nuevas reflexiones e investigaciones semánticas de naturaleza sincrónica en el ámbito de la LEXPED, lo que podrá, sin duda, ayudar para que tengamos cada vez más resultados investigativos coherentes con los principios de la Lexicografía Pedagógica. 


\section{REFERÊNCIAS}

BIDERMAN, M. T. C. Polissemia versus homonímia. In.: Anais do Seminário do Gel XXXVIII, Franca: Unifran - União das Faculdades Franciscanas, 1991.

BIDERMAN, M. T. C.. A ciência da Lexicografia. In.: BIDERMAN, M. T. C. Lexicologia e Lexicografia. Alfa, 28, 1984. (Suplemento): 1 - 26.

BIDERMAN, M. T. C. Dicionário Didático de Português. 2. ed. São Paulo: Ática, 1998.

CASTILLO CARBALLO, $\mathrm{M}^{\mathrm{a}} \mathrm{A}$. La macroestructura del diccionario. In.: MEDINA GUERRA, A. M. (coord.). Lexicografía española. Barcelona: Editorial Planeta, S. A., 2003.

CLAVERÍA, G.; PLANAS, C. La homonimia en la lexicografía española. In.: Nueva revista de filología hispánica. T. 49. № 2, 2001.

CUNHA, A. G. da. Dicionário etimológico da língua portuguesa. 4. ed. Revista pela nova ortografia. - Rio de Janeiro: Lexikon, 2010.

FERNÁNDEZ-SEVILLA, J. Problemas de lexicografía actual. Bogotá: Instituto Caro e Cuervo, 1974.

GONZÁLEZ, M. C. Diccionario de español para extranjeros - con el español que se habla hoy en España y en América Latina. Coordinación y proyecto editorial Concepción. São Paulo: edições SM, 2005.

HARMANN, R. R. K. On theory and practice - theory and practice in dictionarymaking. In: HARMANN, R. R. K. (Org.). Lexicography: principles and practice. Longon: Academic Press Inc., 1983.

KRIEGER, M. da G. In: XATARA, C.; BEVILACQUA, C. R.; HUMBLÉ, P. R. (Orgs). Dicionários na teoria e na prática: como e para quem são feitos. São Paulo: Parábola Editorial, 2011.

LYONS, J. Linguagem e linguística: uma introdução. Rio de Janeiro: LTC, 1987.

MOLINA, D. Fraseología bilingüe: un enfoque lexicográfico-pedagógico. Granada: Editorial COMARES, 2006.

MURAKAWA, C. de A. A.; ZAVAGLIA, C.. Questões teóricas específicas. In.: XATARA, C.; BEVILACQUA, C. R.; HUMBLÉ, P. R. M. Dicionários na teoria e na prática: como e para quem são feitos. São Paulo: Parábola, 2011.

PEREIRA, R. R. O dicionário pedagógico e a homonímia: em busca de parâmetros didáticos. Tese (Doutorado em Linguística e Língua Portuguesa). Universidade Paulista 
“Júlio de Mesquita Filho", Faculdade de Ciências e Letras: Araraquara, 2018, 209 p.

PEREIRA, R. R.; NADIN, O. L. Bases teóricas e metodológicas para o inventário de candidatos a homônimos destinados a dicionários pedagógicos. Revista Signótica, v. 32, 2020 .

RUNDELL, M. Recent trends in English pedagogical lexicography. International Journal of Lexicography, 11, 4, 1998.

SÁNCHEZ RAMOS, M. del M. Tipología lexicográfica en la enseñanza del inglés como segunda lengua: hacia una descripción del uso de los diccionarios. Odisea, n. 4, 2003. Disponível em: http://www.ual.es/odisea/Odisea04_SanchezRamos.pdf. Acesso em: 02 de fevereiro de 2016.

PORTO DAPENA, J. Manual de técnica lexicográfica. Madrid: ARCO/LIBROS, S. A., 2002.

ULLMANN, S. Semântica: uma introdução à ciência do significado. Tradução de J.A. Osório Mateus. Lisboa: Fundação Calouste Gulbenkian, 1964.

WERNER, R. Lexico y teoria general del lengage. In: HAENSCH, G. et al. $L a$ Lexicografia. De la Linguística teórica a la Lexicografia práctica. Madrid: Editorial Gredos: 20- 94, 1982.

WELKER, H. Lexicografia Pedagógica: definições, história, peculiaridades. In: XATARA, C.; BEVILACQUA, C.; HUMBLÉ, P. Lexicografia Pedagógica: pesquisas e perspectivas. Santa Catarina: NUT, 2008.

ZAVAGLIA, C. Ambiguidade gerada pela homonimia: revisitação teórica, linhas limítrofes com a polisemia e propostta de criterios distintivos. DELTA: Documentação de Estudos em Linguísta Teórica e Aplicada, São Paulo, v. 1, n. 19, 2003, p. 337 - 266.

ZAVAGLIA, C.; MURAKAWA, C. de A. A. Questões teóricas específicas. In.: XATARA, C.; BEVILACQUA, C. R.; HUMBLÉ, P. R. M. Dicionários na teoria e na prática: como e para quem são feitos. São Paulo: Parábola, 2011. 